\begin{tabular}{|c|c|}
\hline 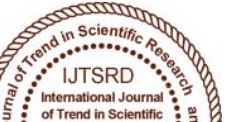 & $\begin{array}{l}\text { International Journal of Trend in Scientific Research and Development (IJTSRD) } \\
\text { International Open Access Journal | www.ijtsrd.com }\end{array}$ \\
\hline 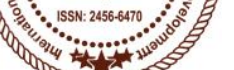 & ISSN No: 2456 - 6470 | Volume - 2 | Issue - 6 | Sep-Oct 2018 \\
\hline
\end{tabular}

\title{
Non Linear Analysis of Diesel Engine Connecting Rod
}

\author{
Vytla Jayaprakash $^{1}$, Dr. Alok Kumar Rohit ${ }^{2}$, Ch. Srinivas ${ }^{3}$ \\ ${ }^{1}$ M.Tech, ${ }^{2}$ Professor, ${ }^{3}$ B.Tech \\ Mechanical Department, Jain University, Karnataka, India
}

\begin{abstract}
the connecting rod is the mediator member between the piston and the Connecting Rod. Its primary function is to transmit the push and pull from the piston pin to the crank pin and thus convert the reciprocating motion of the piston into rotary motion of the crank. For piston pin there will be tiny end is fix, it will used mostly to press fit into the connecting rod but it can swivel in the piston, a "floating wrist pin" design. Presently connecting rod is manufacturing by using the material Carbon steel. In this project, connecting rod is designed using Aluminum alloys 7475 and 6061. The aim of this project is to design the connecting rod for $150 \mathrm{cc}$ engine motorbike by using Design formulas for the above materials. By using catia software we can draw the 3Dmodel of the connecting rod as per the parameters. All the required data is collected from design data books, internet and journals.
\end{abstract}

Keywords: Connecting rod, ansys, static analysis

\section{INTRODUCTION}

As we know the reciprocating piston engine, the connecting rod affixes the piston to the crankshaft. In the modern automobiles industries the connecting rods is made up of steel for manufacturing engines, but it can be made of aluminum (for light in weight and the aptness to soak up the vast knock at the expense of durability) or with titanium also we can construct (for a amalgamation of power and lightness at the costly of affordability) for high production engines. They are not stiffly fixed at either end, so that the angle between the connecting rod and the piston can transmogrify as the rod goes up and down and twirl around the crankshaft. Condors', particular in racing engines, may be known as "billet" rods. The tiny end affix to the piston pin, gudgeon pin (the usual British term) or wrist pin, which is presently most frequently press fit into the connecting rod but can pivot in the piston, a "floating wrist pin" design. The vast end affix to the bearing journal on the crank throw, moving on replaceable bearing shells available via the connecting rod bolts which grasp the bearing "cap" onto the vast end; typically there is a pinhole bored through the bearing and the vast end of the connecting rod so that pressurized lubricating motor squirts out onto the thrust of the cylinder wall to lubricate the travel of the pistons and piston rings.

\section{METHODS GENERALLY USED FOR MANUFACTURING THE CONNECTING ROD Forging Vs Casting
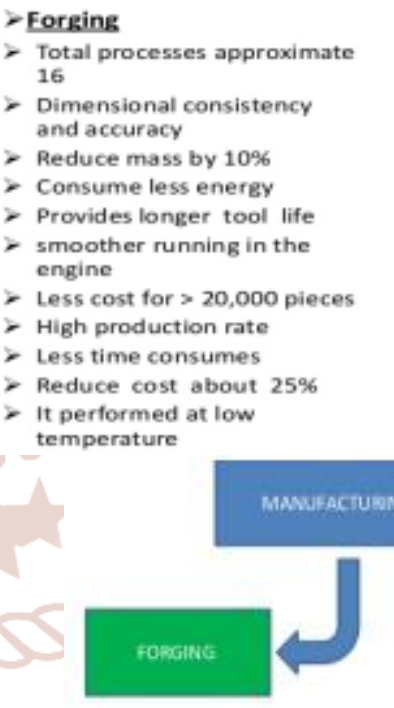

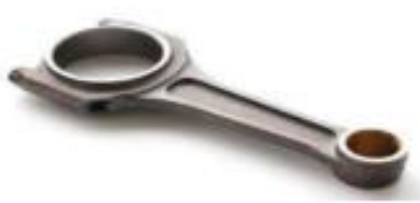

Fig .1, Manufacturing process

\section{INTRODUCTION TO CAD}

As we know to design of an object in computer is difficult in computer in past days but now a day's it's 
so simple firstly we know Computer-aided design (CAD) it is software now a day's it's made so easy to draw $2 \mathrm{D}$ and $3 \mathrm{D}$ diagrams in computers. It's also called as computer-aided design and drafting (CADD).By the using of Computer Aided Drafting which explains the drafting process in computer. For many of design engineering CADD software is a flagship tool by using this software they shows the elegant designs of the objects as per the requirements. The output of CADD is in the form of print or machining operations.

The required output information is also important for CAD such as materials, processes, dimensions, and tolerances, according to specific applications. The design curves and figures in two-dimensional (2D) space and solids surface in three-dimensional (3D) objects are shown in CAD software.. The design of geometric objects for object shapes, in particular, is often called computer-aided geometric design (CAGD).

\section{ANSYS - AN OVERVIEW}

For design and investigation of engineering difficulties or problems we can solve by using the software is called ANSYS software. The ANSYS is finite element analysis software for advanced by ANSYS INC. It is user friendly graphical user interface package. Many no of CAD Programmers have straight interfaces with the ANSYS program through software written by ANSYS.INC or by the $\mathrm{CAD}$ vendors. Interpreter for the programs like AutoCAD and Pro/Engineer are accessible from ANSYS.INC.

There are following tasks which enable the ANSYS finite element analysis software for engineers to execute the performance on the models.

1. Construct the computer models or send CAD models of structures, products, components or system.

2. Petition the operating loads or other design production state.

3. Examine the physical properties, such as stress levels, temperature disseminate, etc.

4. Optimize a design early in growth action to diminish manufacture prices.

5. Do prototype testing in ambient where it otherwise would be undesirable or impossible.

6. The essential goal of finite element analysis is to investigate how the responds from the elements or models under the certain loading condition

\section{PROCESS FOR ANSYS ANALYSIS}

By utilizing the Static analysis we can find out the displacements, stresses, strains and forces in structures or components during the loads conditions that do not leading remarkable inertia and damping effects. Firstly Steady loading conditions are to be assumed. These types of loading that can be applied in a static analysis include the forces and pressures, steady state inertial forces such as gravity velocity imposed (non zero) displacements, temperature (for thermal strain). A static analysis can be either linear or non linear. For your work we are choosing the linear statistic analysis. For Procedure of static analysis it consists 3 main steps they are:

$>$ Construction of the model

Getting the solution.

Reviewing the repercussion.

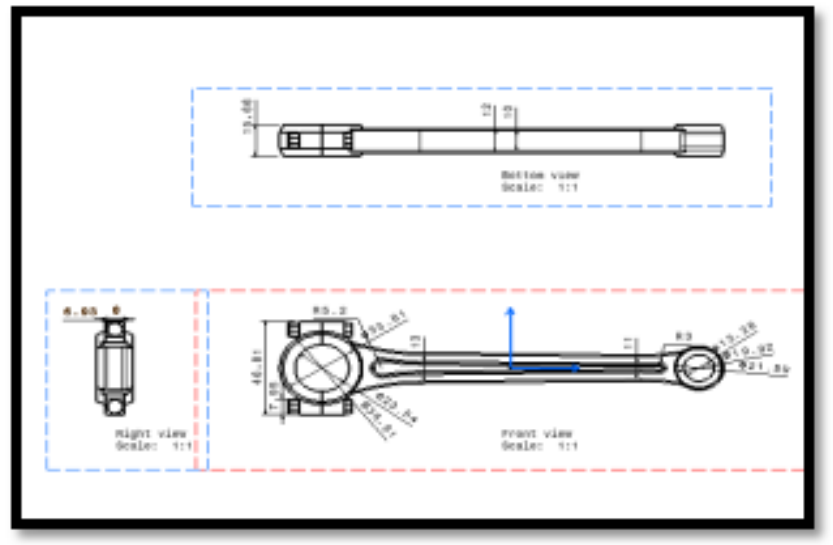

Fig.2, 2D MODELOF CONNECTING ROD

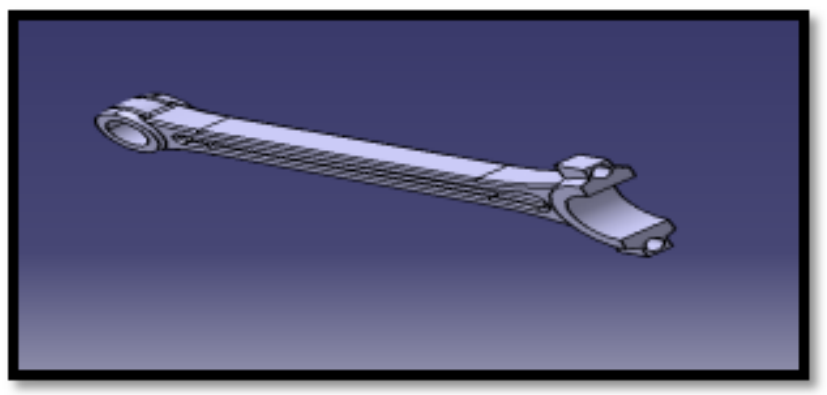

Fig.3, 3D MODEL OF CONNECTING ROD

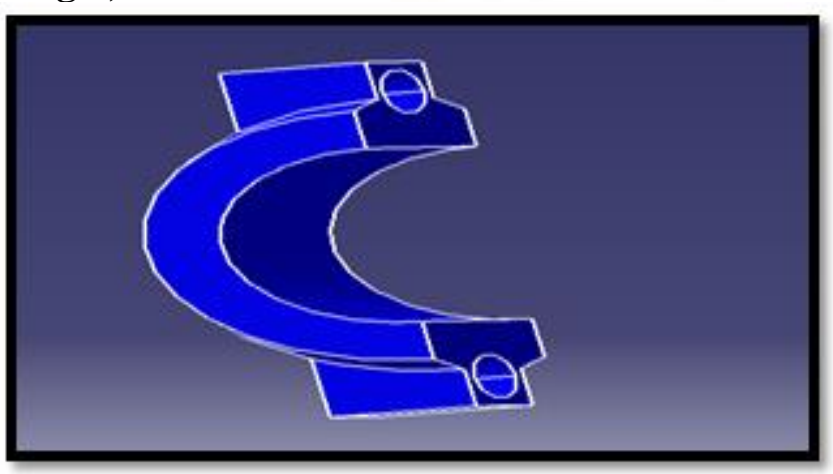

Fig.4, BIG CAP END 


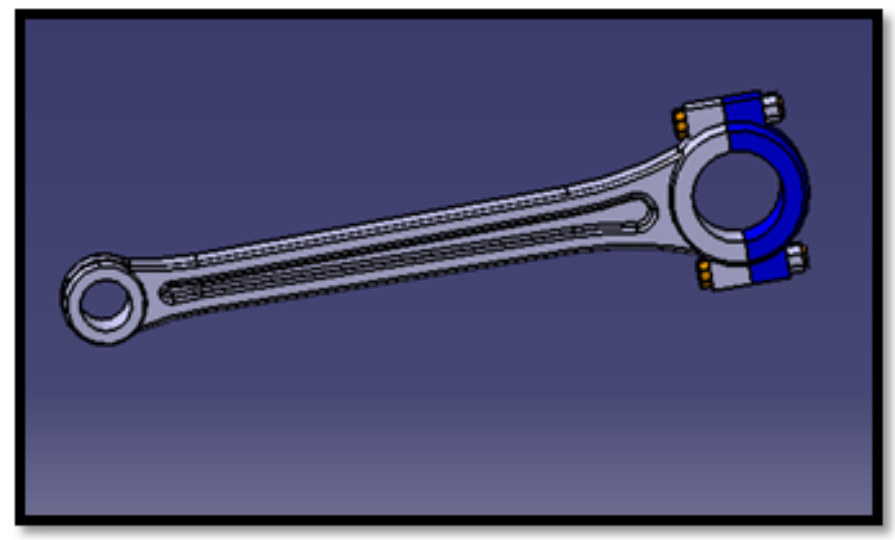

Fig.5, FINAL ASSEMBLY COMPONENT

ANALYSIS OF CONNECTING ROD STATIC ANALYSIS

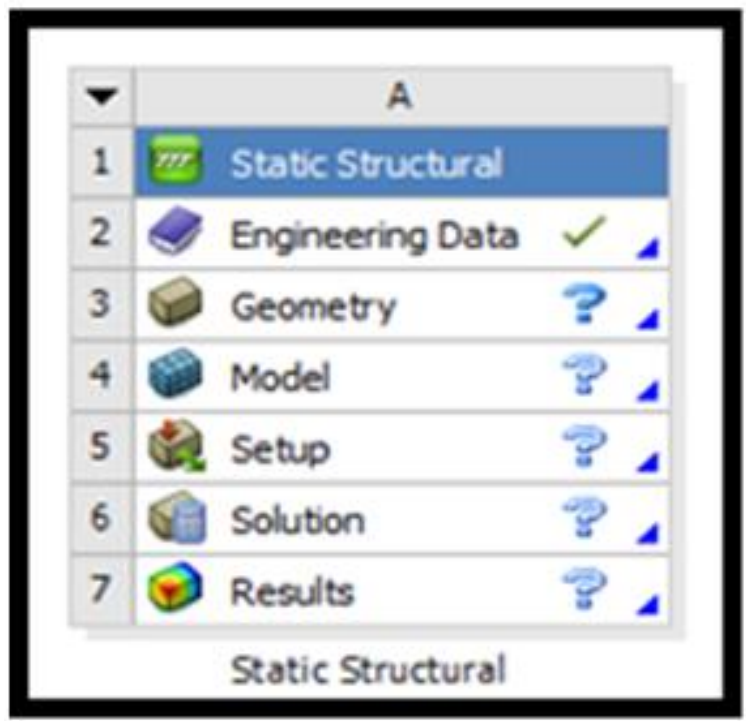

Select engineering data> window will be open in that enter required material properties>

\section{MATERIAL - ALUMINIUM ALLOY 7475}

Material properties

Density: $2.81 \mathrm{~g} / \mathrm{cc}$

Young's modulus70.3gpa

Poisson's ratio: 0.33

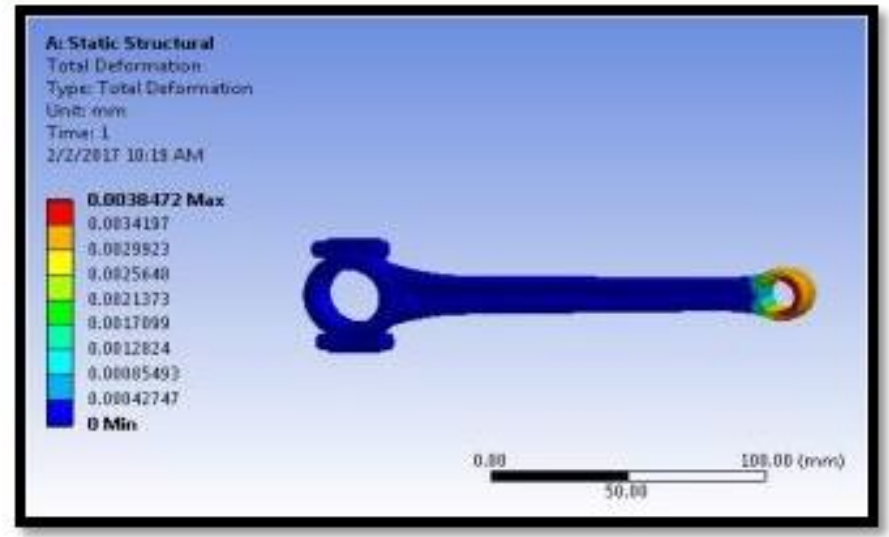

Fig.6, Deformation

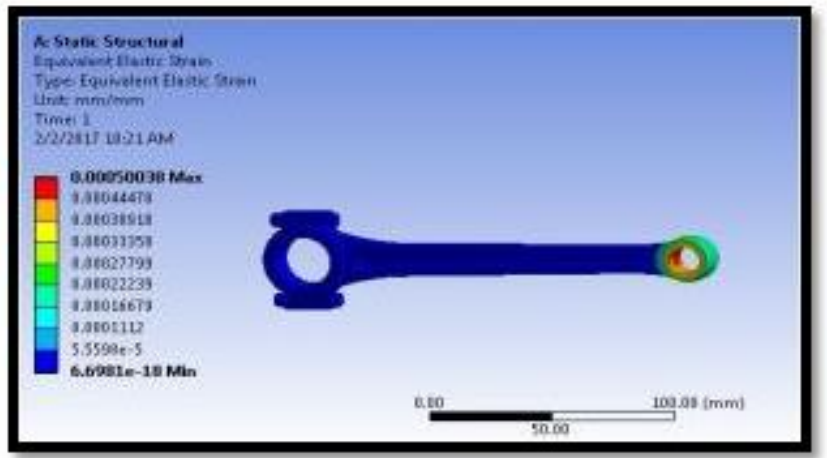

Fig.7, Strain

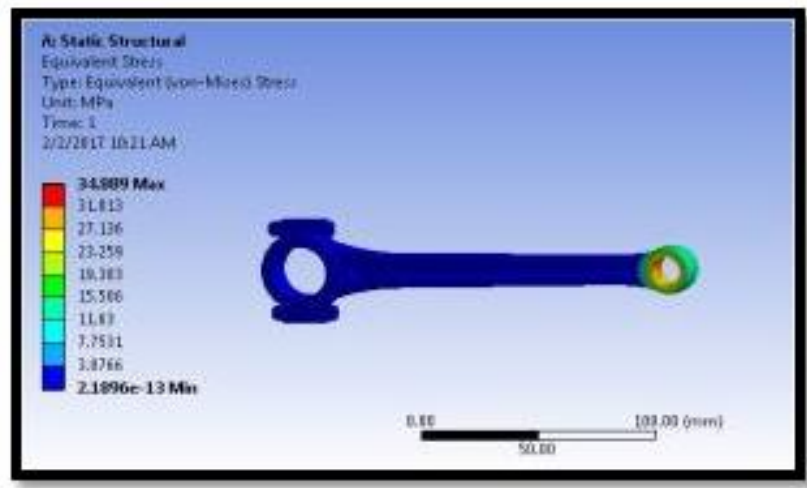

Fig.8, Stress

MATERIAL - ALUMINIUM ALLOY 6061

Material properties

Density: $2.70 \mathrm{~g} / \mathrm{cc}$

Young's modulus68.9gpa

Poisson's ratio: 0.33

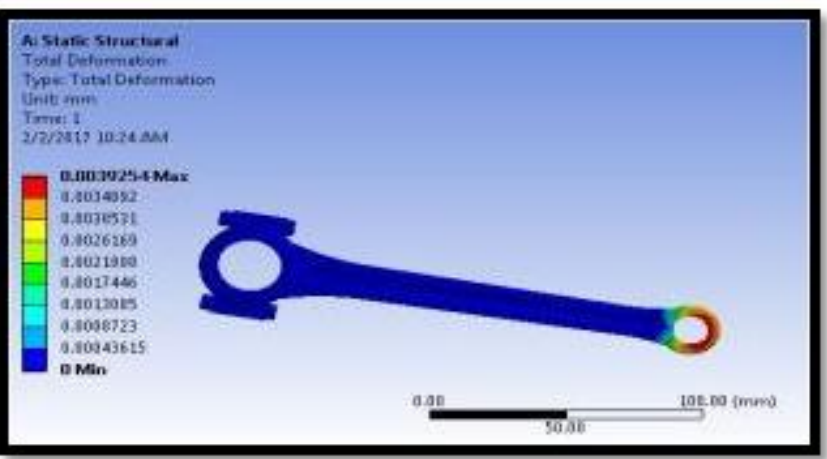

Fig: .9, Deformation

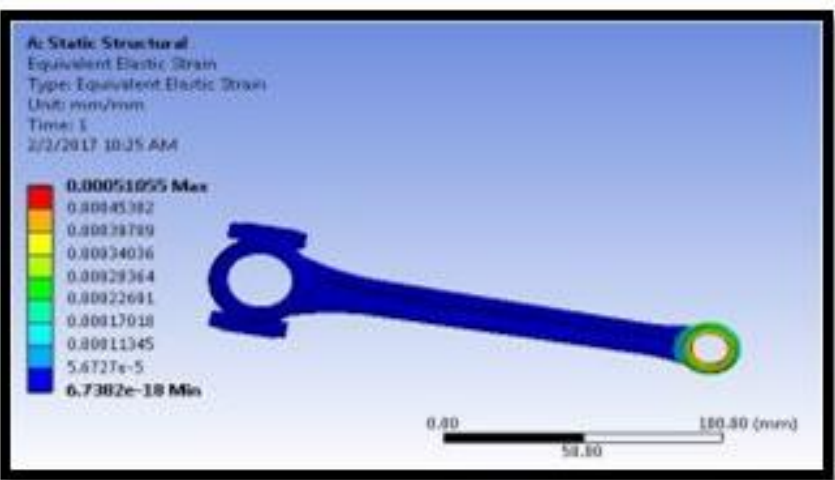

Fig.10, Strain 
International Journal of Trend in Scientific Research and Development (IJTSRD) ISSN: 2456-6470

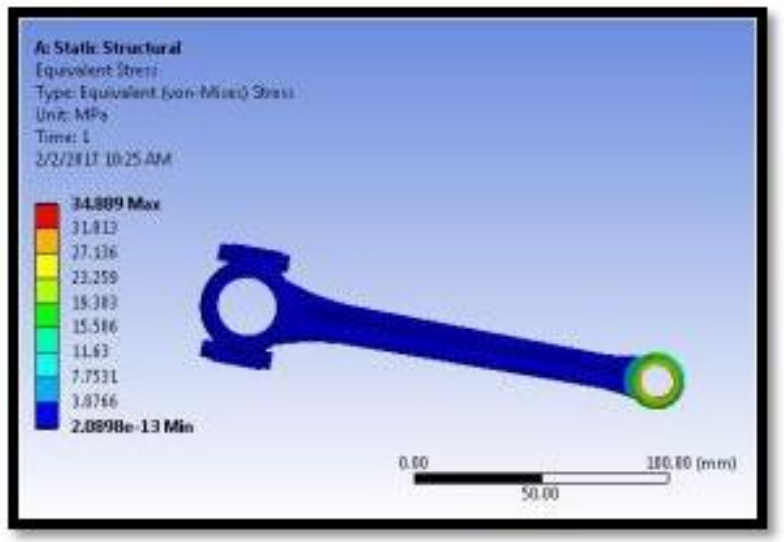

Fig.11, Stress

B. MODAL ANALYSIS CARBON STEEL

ANSYS > Work bench 14.5>Double click on Modal.

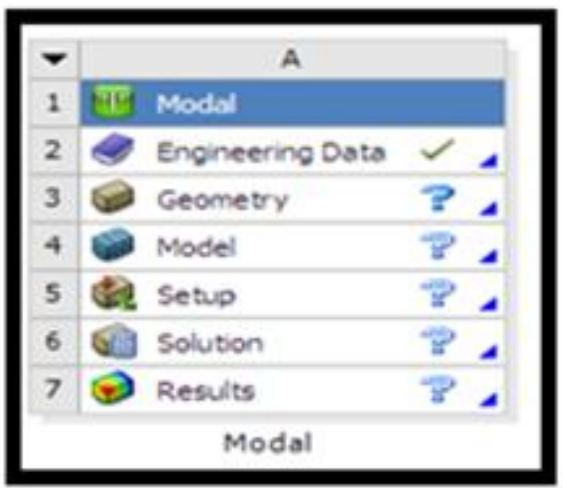

Right click on Engineering data $>$ edit $>$ apply material properties $>$ return project $>$ Update project.

Material properties

Density: $7.89 \mathrm{~g} / \mathrm{cc}$

Young's modulus: $213 \mathrm{GPa}$

Poisson's ratio: 0.30

Right click on Geometry>Imported Geometry>browse>click on IGS file>Open.

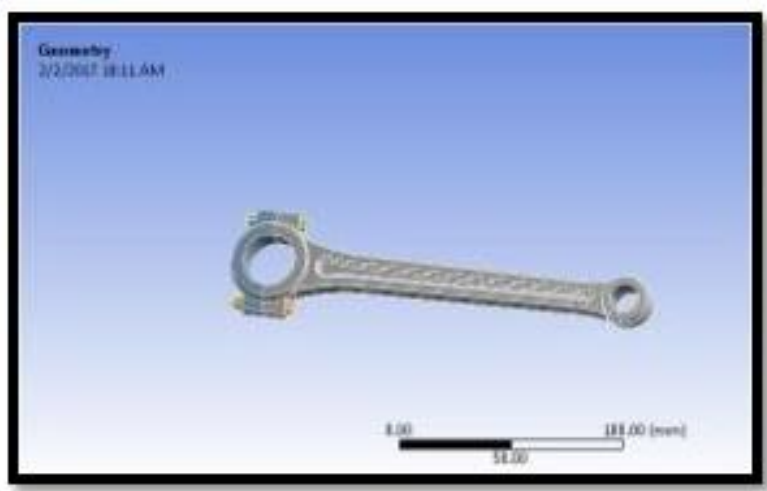

Fig.12, Imported Geometry

Right click on Model $>$ Edit $>$ Right click on mesh>sizing > fine $>$ Right click on mesh>generate mesh.
Right click on Modal $>$ Insert $>$ Displacement $>$ Select faces $>$ apply.

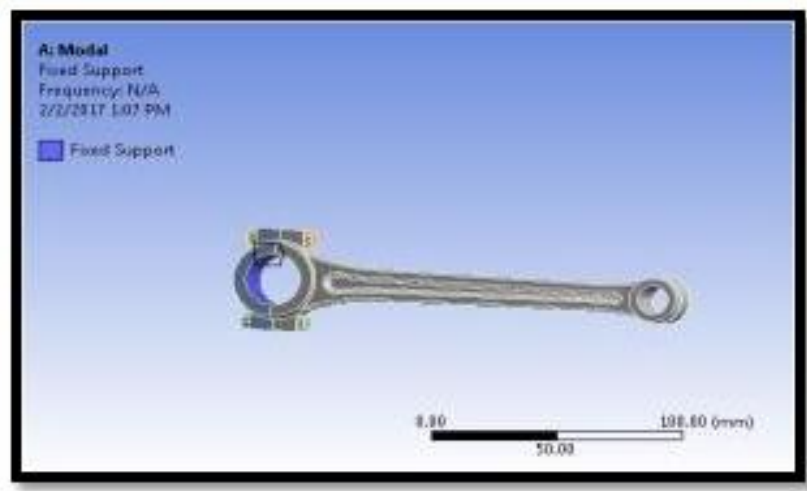

Fig.13, Fixed support

Right click on

Solution $>$ Insert $>$ Deformation $>$ Total $>$ Mode 1 .

Right click on

Solution $>$ Insert $>$ Deformation $>$ Total $>$ Mode 2 .....etc Right click on Solution >Solve.

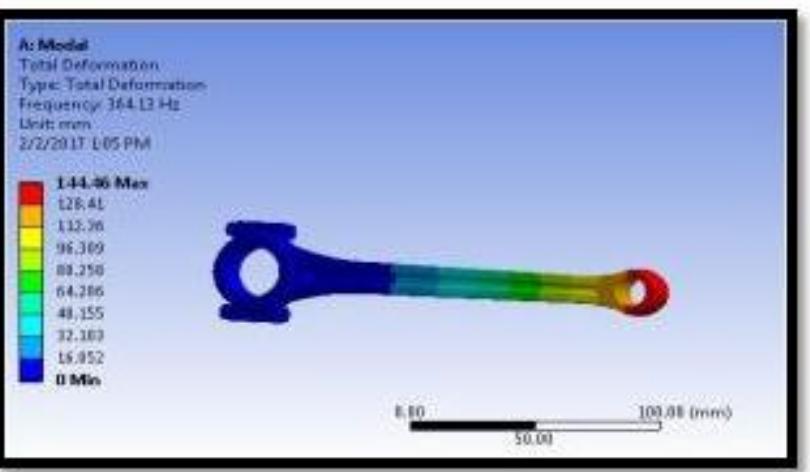

Fig.14, Mode 1

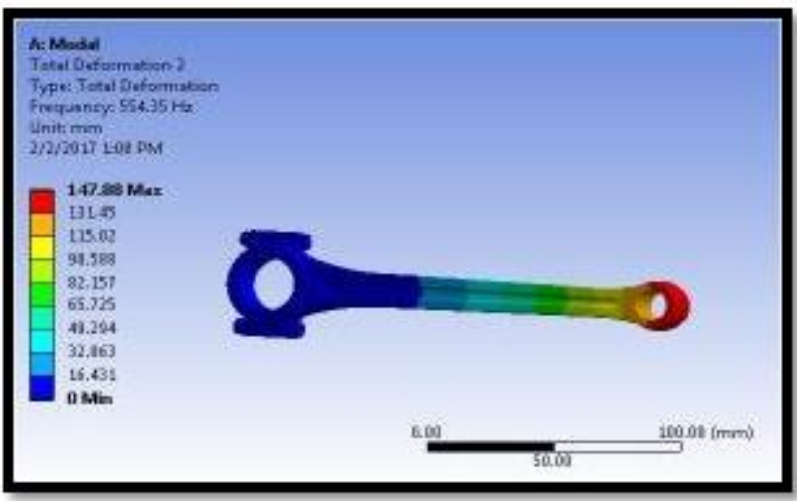

Fig.15, Mode 2

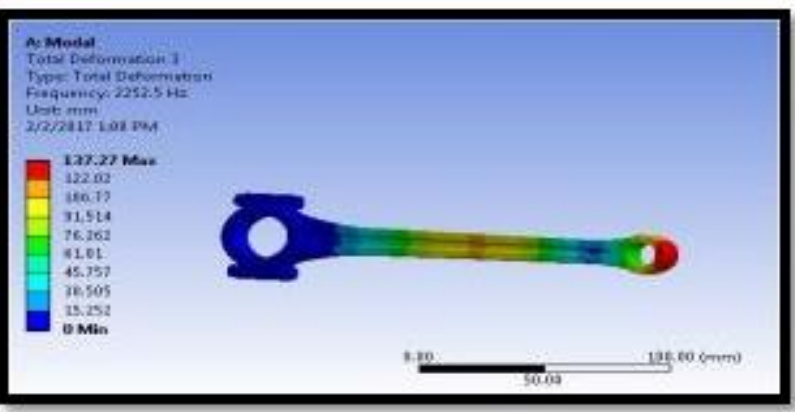

Fig.16, Mode 3 
International Journal of Trend in Scientific Research and Development (IJTSRD) ISSN: 2456-6470

ALUMINIUM ALLOY 7475

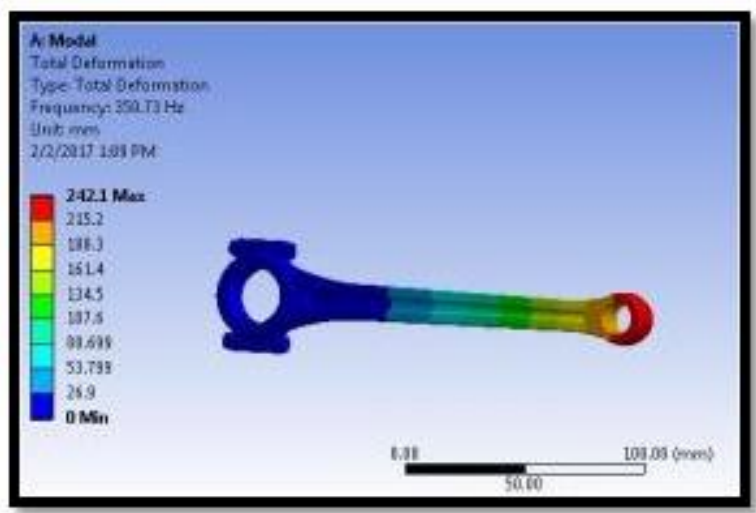

Fig.17, Mode 1

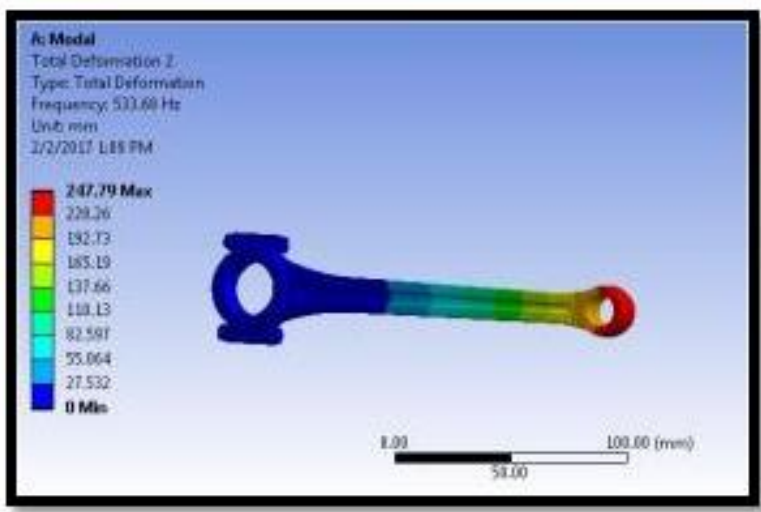

Fig.18, Mode 2

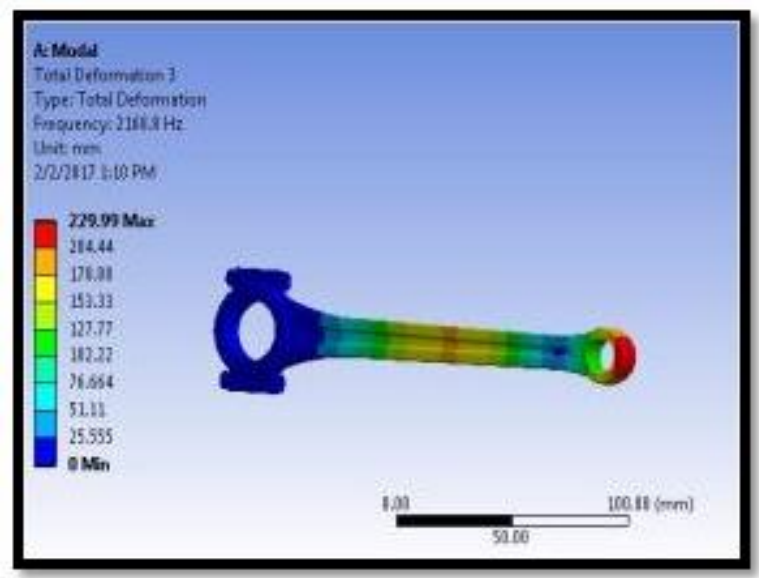

Fig.19, Mode 3

\section{HARMONIC ANALYSIS} MATERIAL - CARBON STEEL

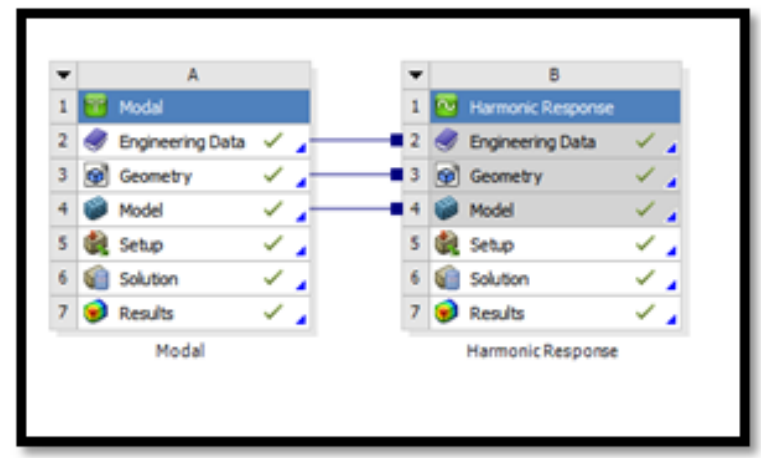

Fig.20, Harmonic Analysis

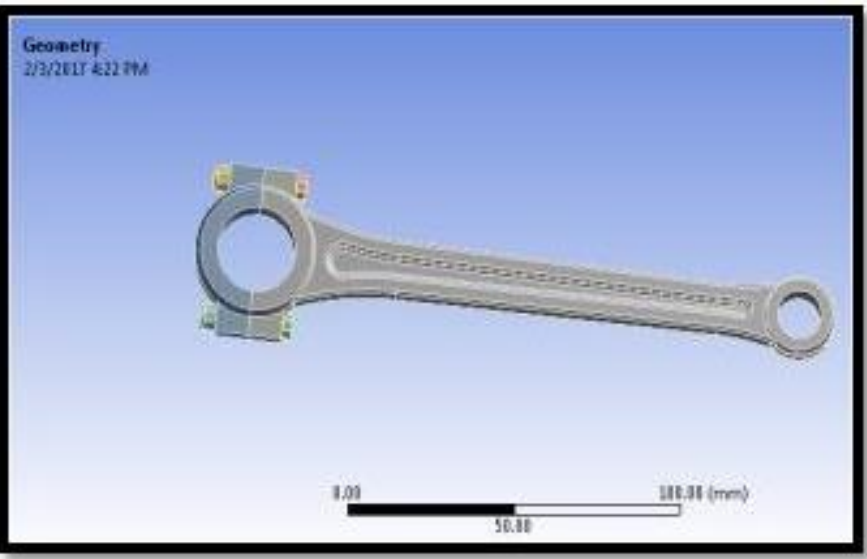

Fig.21, Imported model

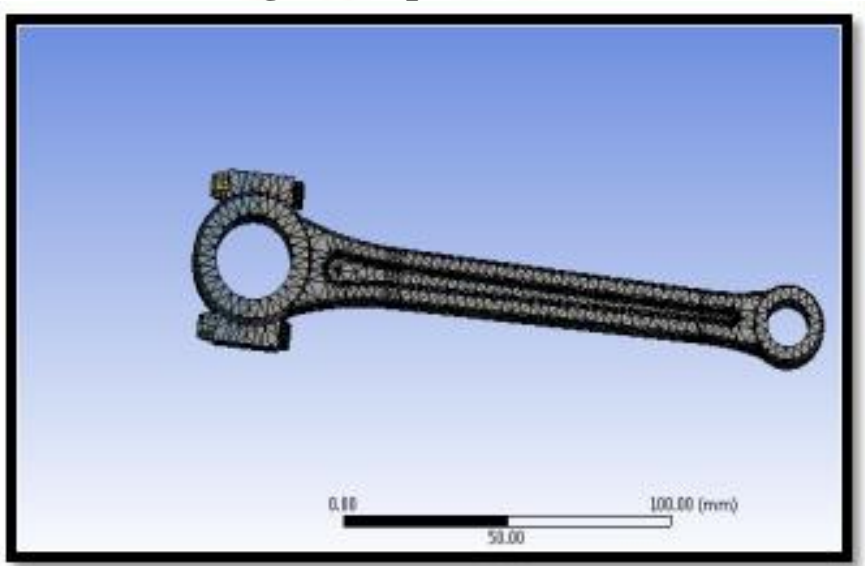

Fig.22, mesh

Enter the higher frequencies value (taken from modal analysis)

\begin{tabular}{|c|c|c|}
\hline \multicolumn{3}{|c|}{ Details of "Analysis Settings" } \\
\hline \multirow[t]{8}{*}{$=$} & \multicolumn{2}{|l|}{ Options } \\
\hline & Range Minimum & 0. $\mathrm{Hz}$ \\
\hline & Range Maximum & $2252.5 \mathrm{~Hz}$ \\
\hline & Solution Intervals & 10 \\
\hline & Solution Method & Mode Superposition \\
\hline & Cluster Results & No \\
\hline & Modal Frequency Range & Program Controlled \\
\hline & Store Results At All Frequencies & Yes \\
\hline+ & \multicolumn{2}{|l|}{ Output Controls } \\
\hline+ & \multicolumn{2}{|l|}{ Damping Controls } \\
\hline+ & \multicolumn{2}{|l|}{ Analysis Data Management } \\
\hline
\end{tabular}

Select displacement $\rightarrow$ select required area $\rightarrow$ click on apply $\rightarrow$

\section{Displacement}

Select Pressure $\rightarrow$ select required area and enter the pressure value $\rightarrow$ click on apply $\rightarrow$

\section{Pressure}

Solution -right click-solve-select solution -right click -total deformation

Select solution -right click -stress

Select solution -right click -strain

Select solution -right click -Phase response

Select solution -right click-Frequency response 
International Journal of Trend in Scientific Research and Development (IJTSRD) ISSN: 2456-6470

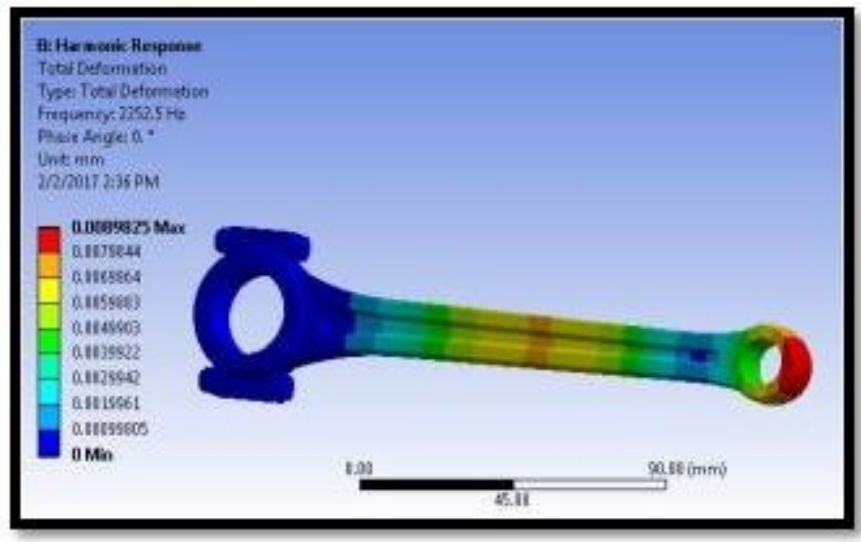

Fig.23, Total Deformation at the frequency

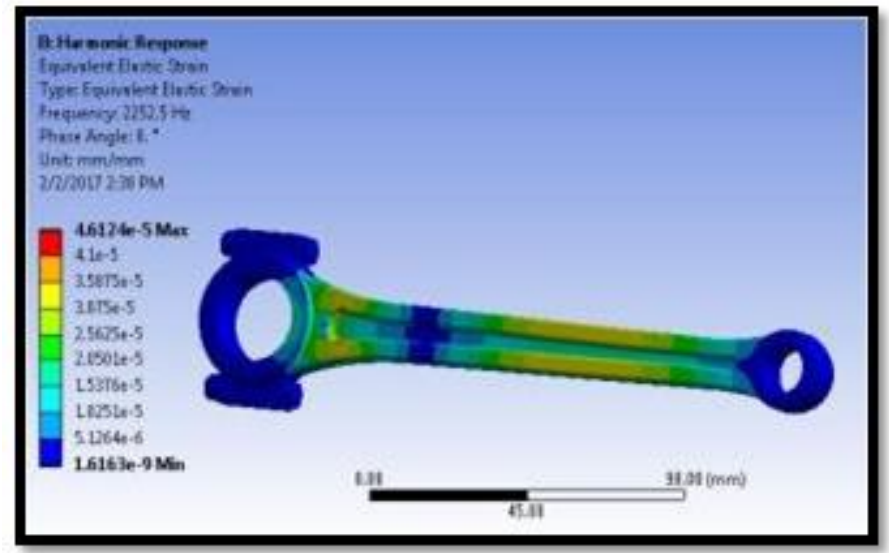

Fig.24, Strain at the frequency

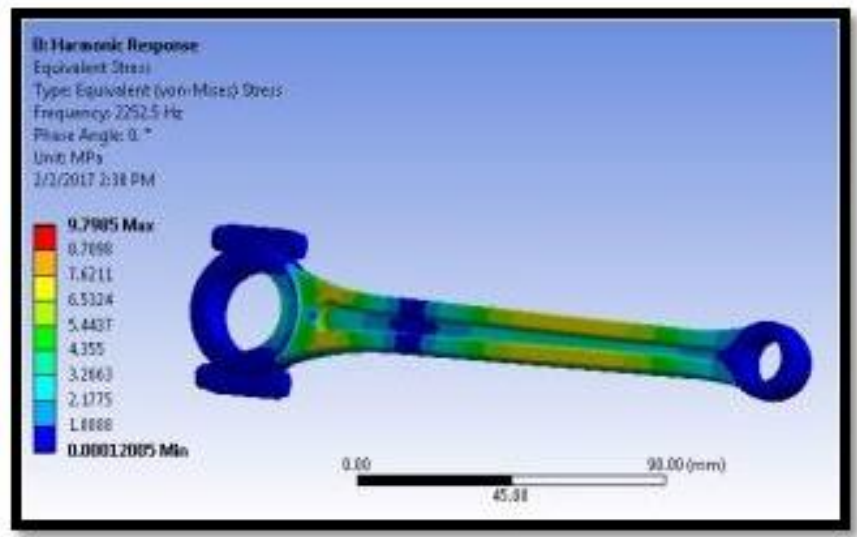

Fig.25, Stress at the frequency

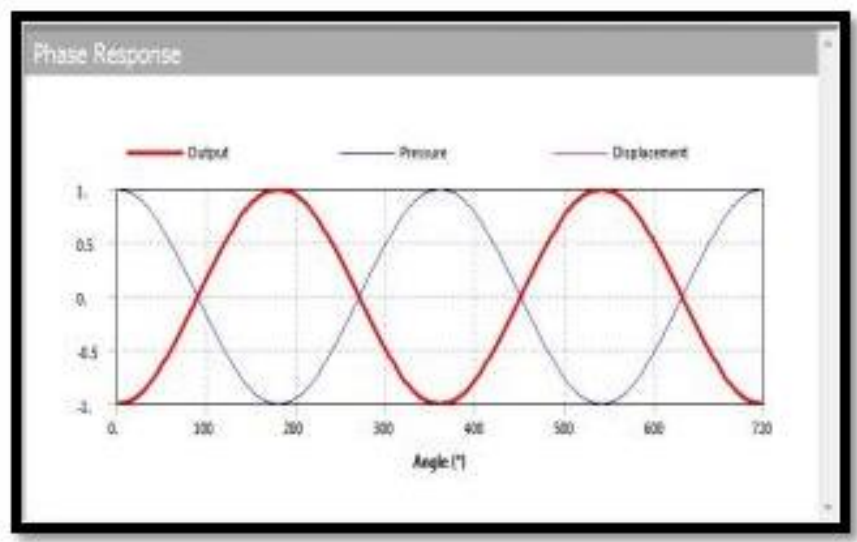

Fig.26, Phase response

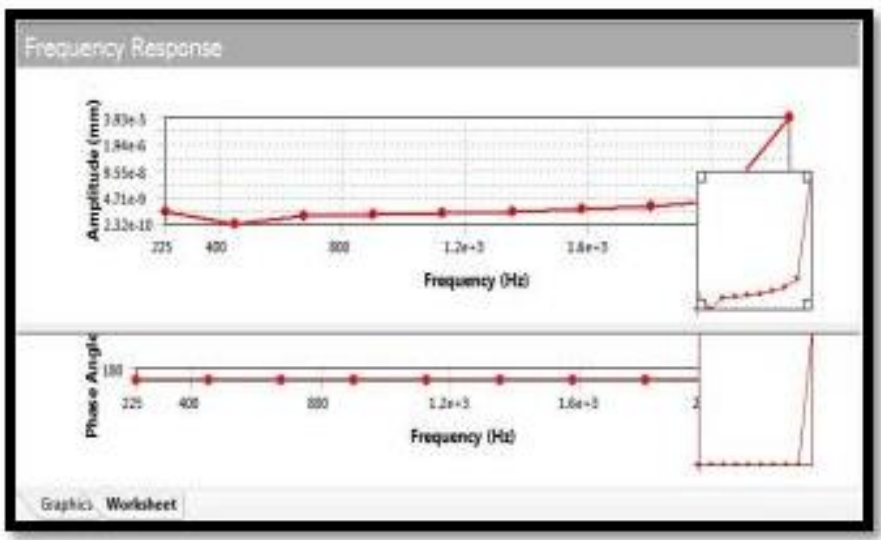

Fig.27, Frequency response

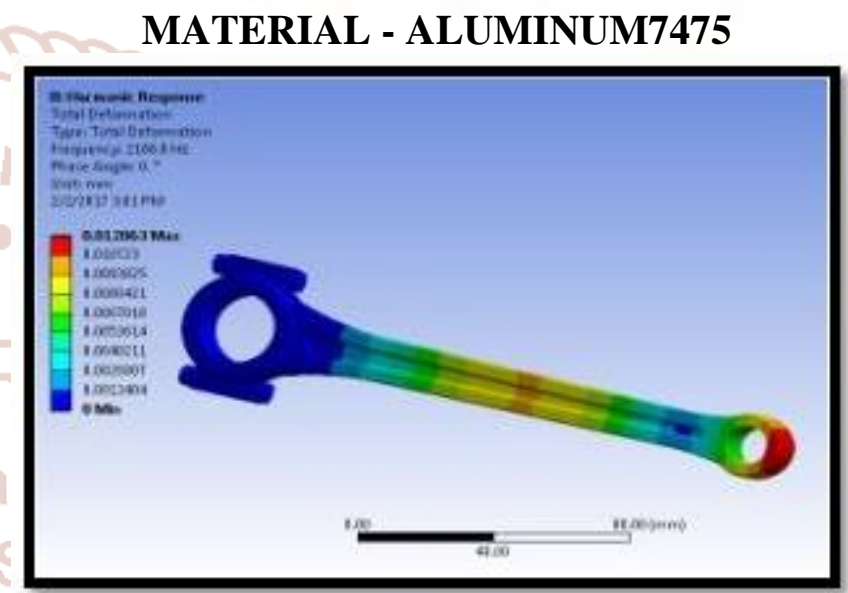

Fig.28, total deformation

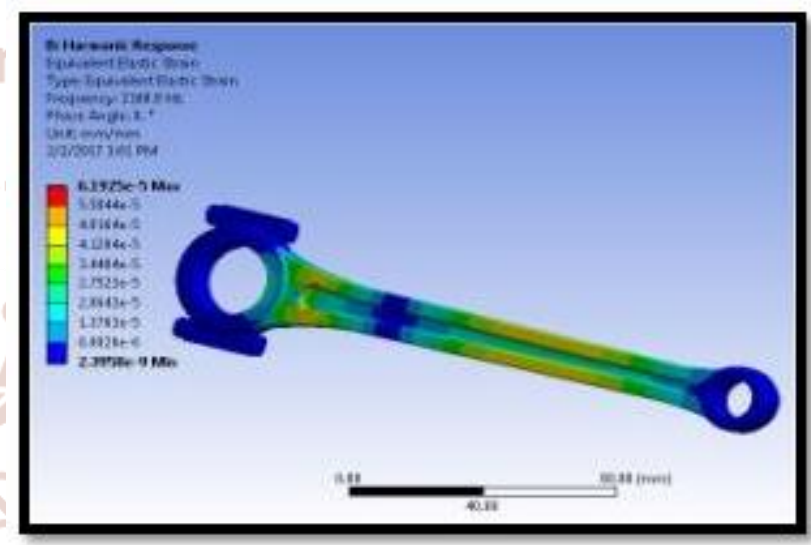

Fig.29, strain

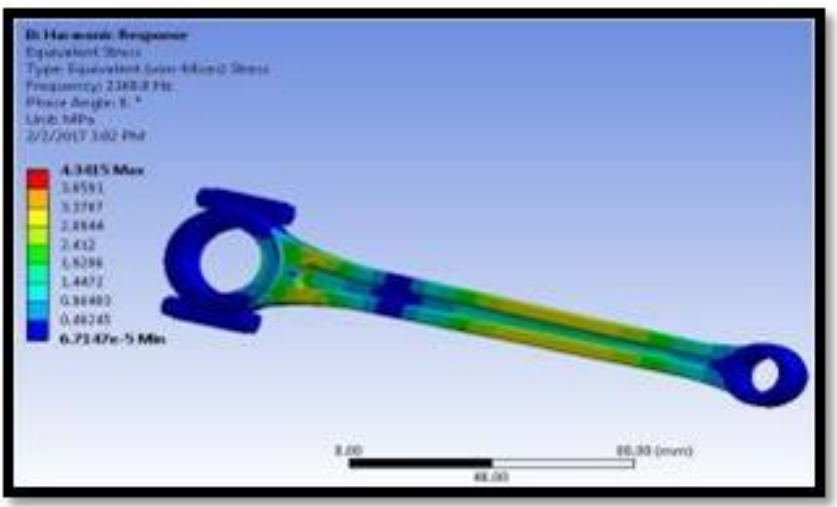

Fig.30, stress 
International Journal of Trend in Scientific Research and Development (IJTSRD) ISSN: 2456-6470

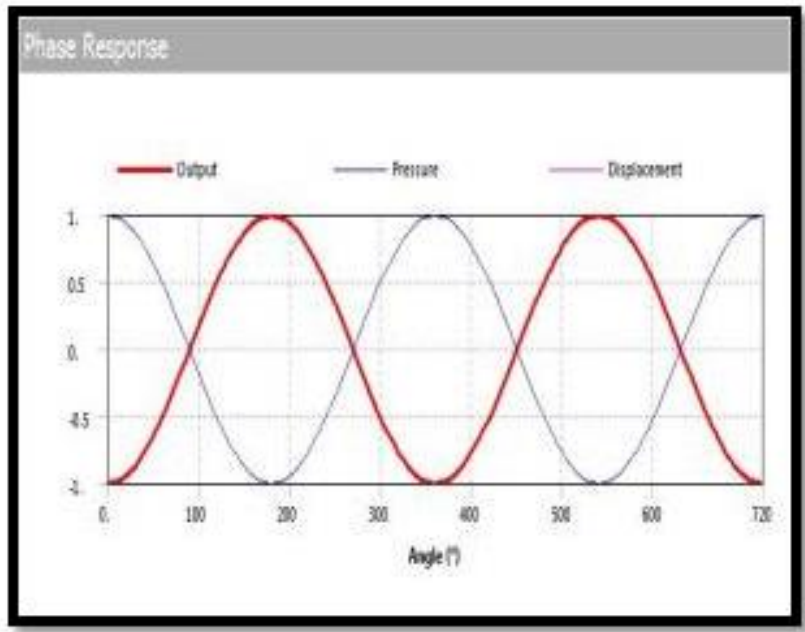

Fig.31, phase response

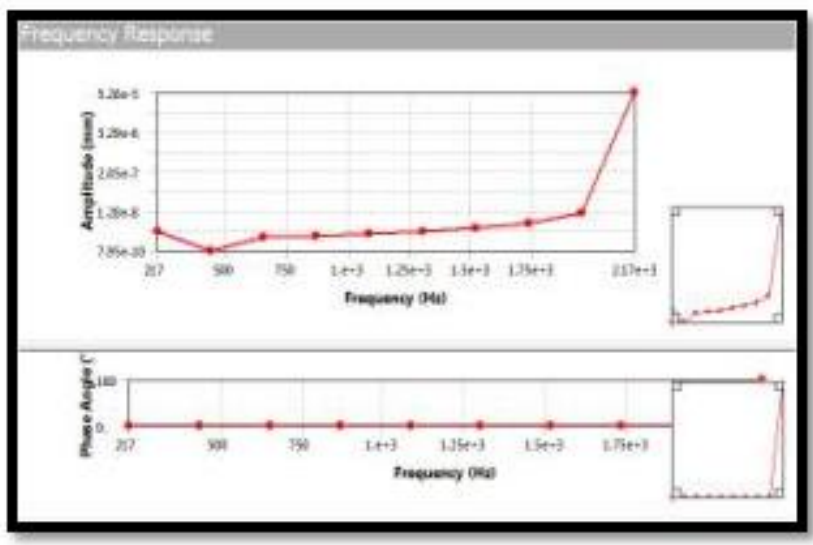

Fig.32, frequency response TRANSIENT STRUCTURAL MATERIAL - Carbon steel At 1sec:

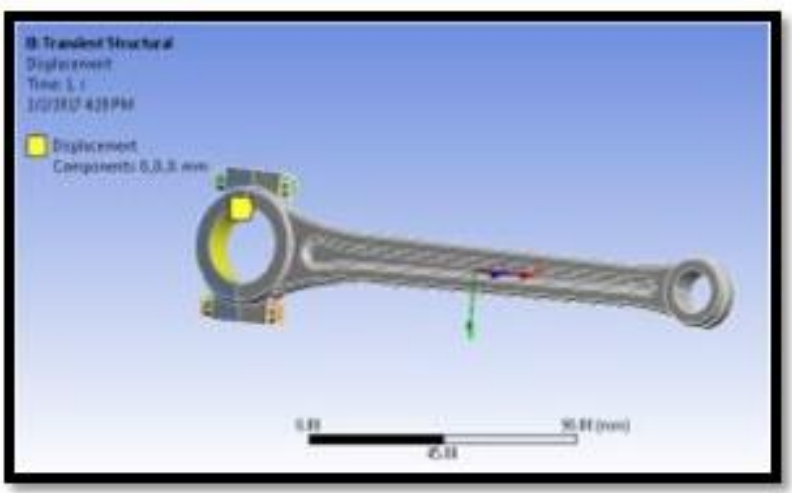

Fig33, displacement

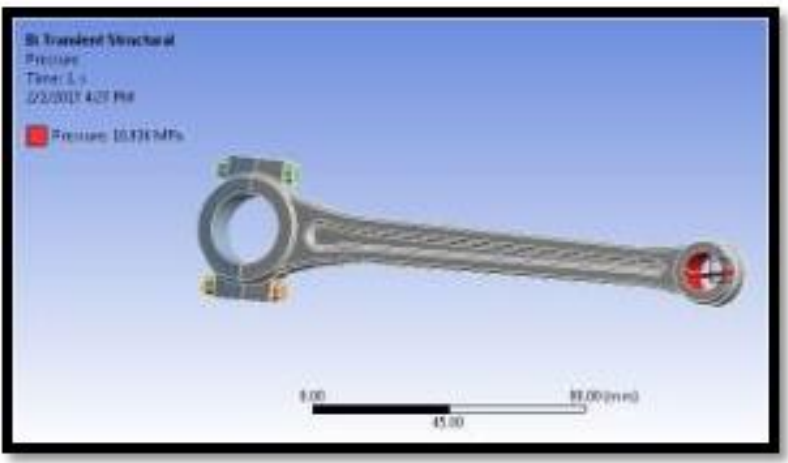

Fig.34, pressure

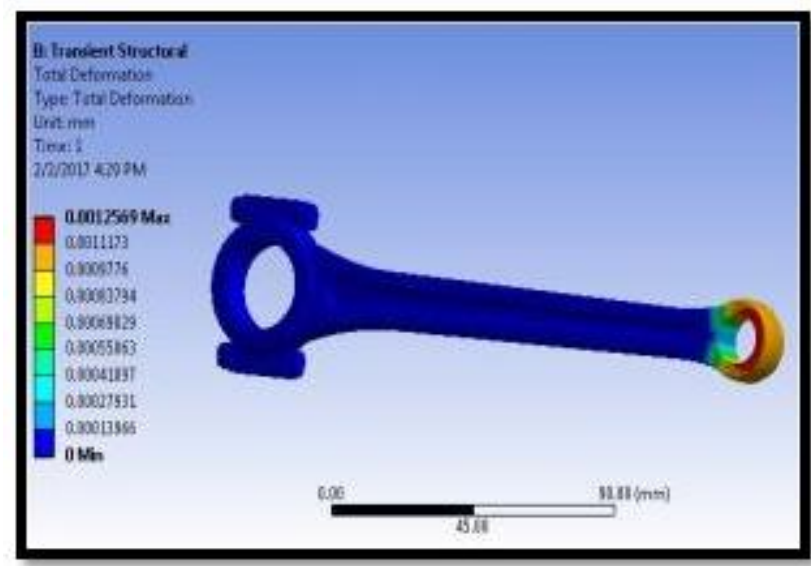

Fig.35, total deformation 5sec:

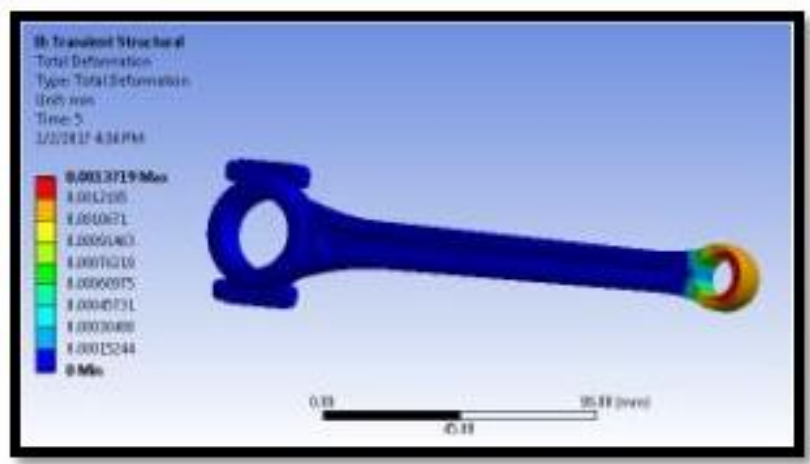

Fig.36, total deformation 10sec

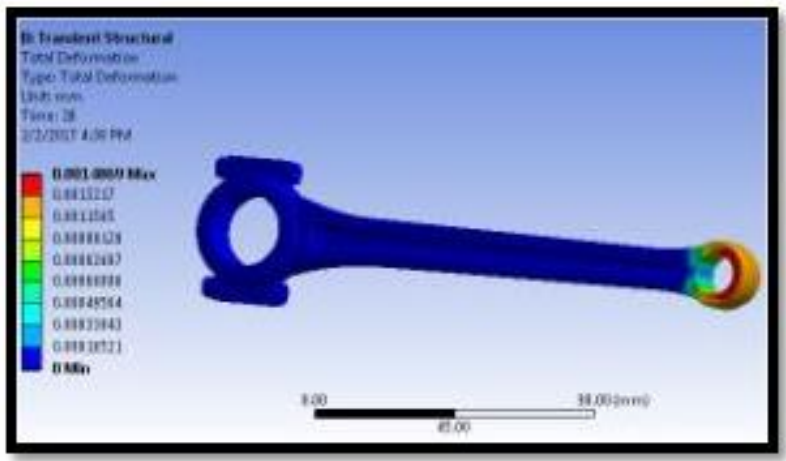

Fig.37, total deformation MATERIAL - ALUMINUM7475 1 sec:

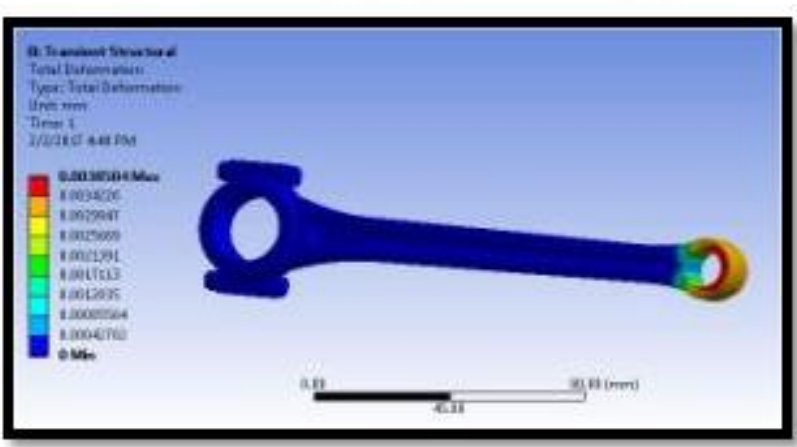

Fig.38, total deformation 5sec 
International Journal of Trend in Scientific Research and Development (IJTSRD) ISSN: 2456-6470

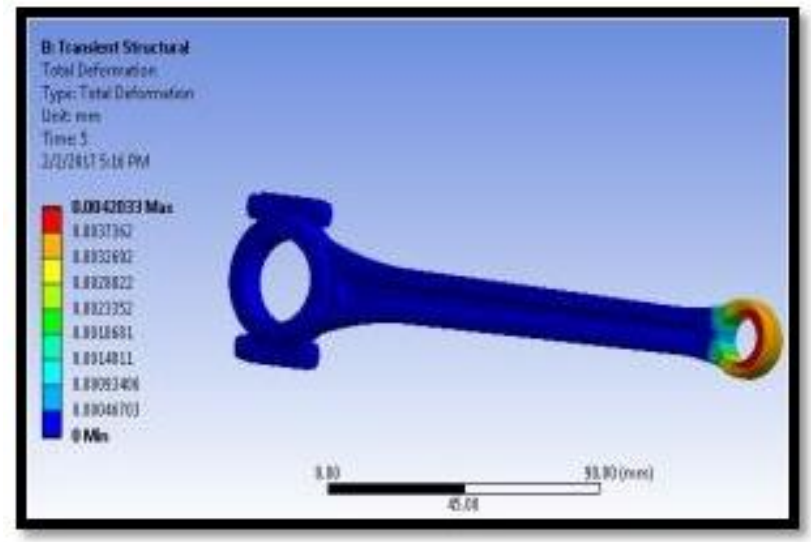

Fig.39, total deformation 10sec:

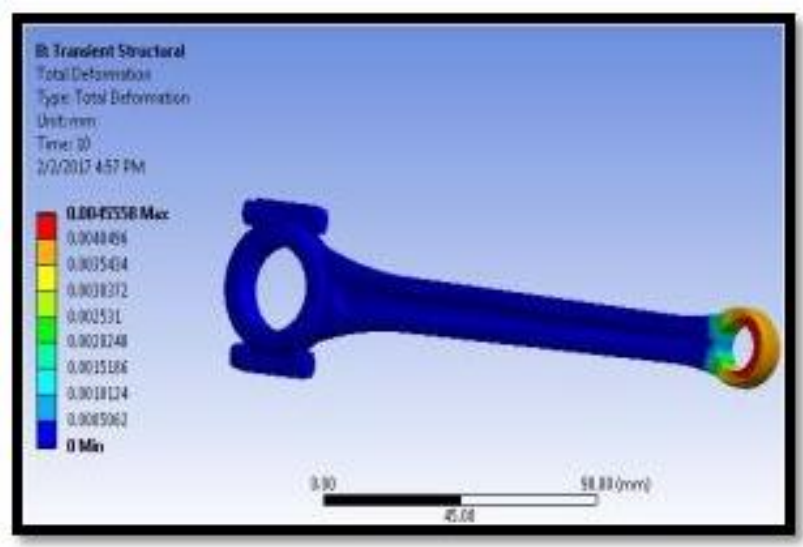

Fig.40, total deformation

\section{RESULT TABLES}

Table.1 STATIC ANALYSIS

\begin{tabular}{|c|c|c|c|}
\hline $\begin{array}{c}\text { Carbon } \\
\text { steel }\end{array}$ & $\begin{array}{c}\text { Deformati } \\
\text { on }(\mathbf{m m})\end{array}$ & Strain & $\begin{array}{c}\text { Stress } \\
\text { (MPa) }\end{array}$ \\
\hline $\begin{array}{c}\text { Aluminum } \\
\text { alloy 7475 }\end{array}$ & 0.0012566 & 0.00016501 & 45 \\
\hline $\begin{array}{c}\text { Aluminum } \\
\text { alloy 6061 }\end{array}$ & 0.0039254 & 0.00050038 & 34.889 \\
\hline
\end{tabular}

GRAPHS.1

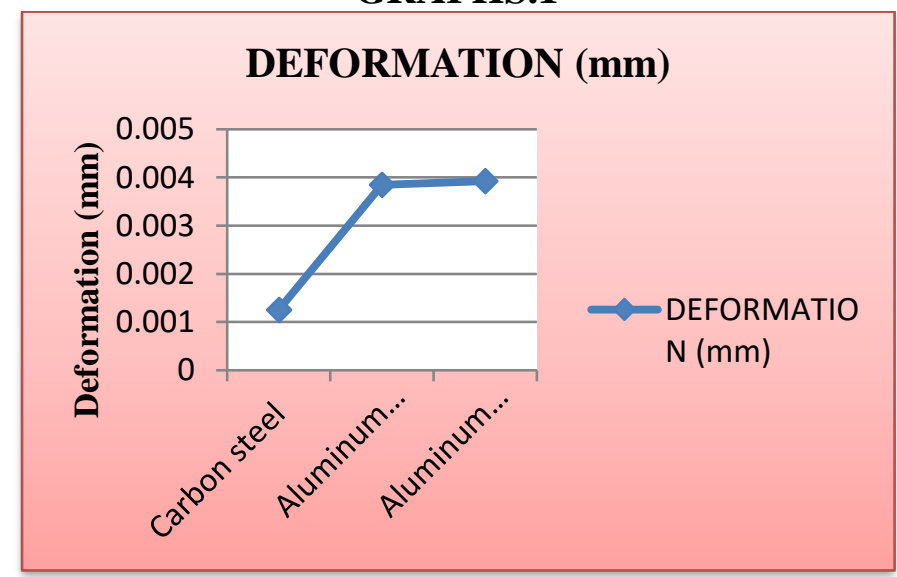

Graph.2

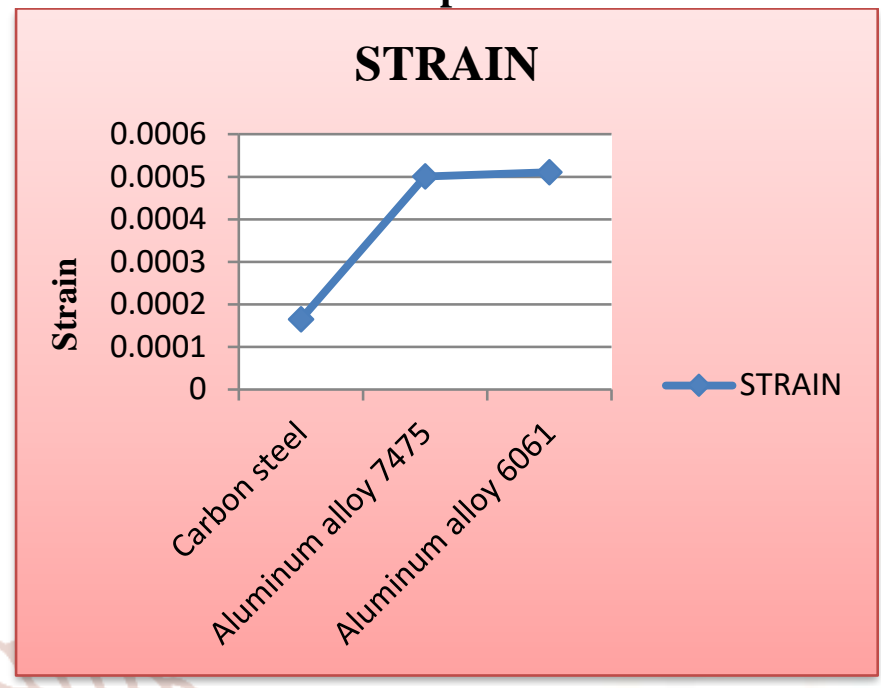

Graph.3

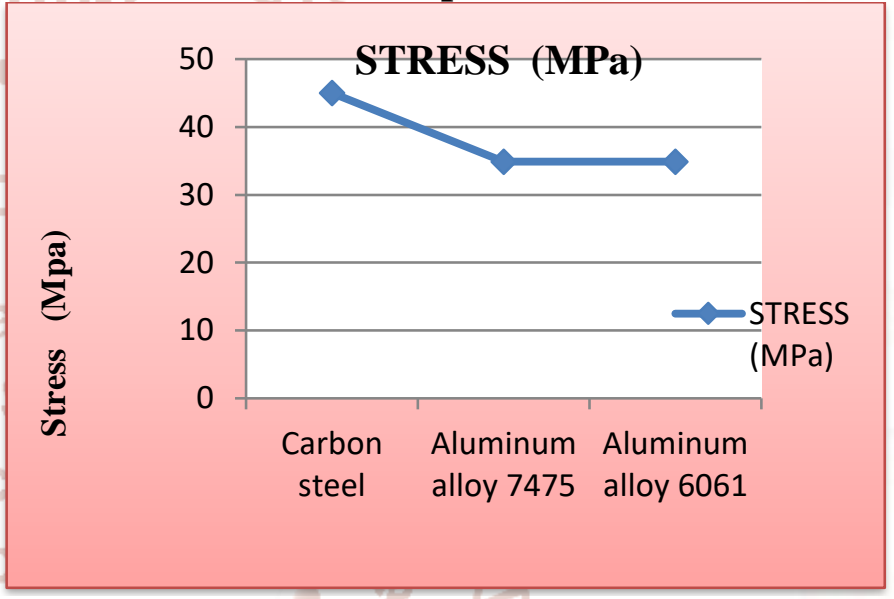

Table .2 MODAL ANALYSIS

\begin{tabular}{|c|c|c|c|}
\hline $\begin{array}{c}\text { Mode } \\
1(\mathrm{~mm})\end{array}$ & $\begin{array}{c}\text { Structura } \\
\text { I steel }\end{array}$ & $\begin{array}{c}\text { Aluminu } \\
\text { m } \\
\text { alloy7475 }\end{array}$ & $\begin{array}{c}\text { Aluminu } \\
\text { m }\end{array}$ \\
\hline $\begin{array}{c}\text { Frequency } \\
1 \text { (Hz) }\end{array}$ & 364.13 & 242.1 & 246.98 \\
\hline $\begin{array}{c}\text { Mode } \\
2 \text { (mm) }\end{array}$ & 147.88 & 247.79 & 252.79 \\
\hline $\begin{array}{c}\text { Frequency } \\
2(\mathrm{~Hz})\end{array}$ & 554.35 & 533.68 & 539.23 \\
\hline $\begin{array}{c}\text { Mode } \\
\text { (mm) }\end{array}$ & 137.27 & 229.99 & 234.63 \\
\hline $\begin{array}{c}\text { Frequency } \\
3 \text { (Hz) }\end{array}$ & 2225.25 & 2168.8 & 2190.4 \\
\hline
\end{tabular}


International Journal of Trend in Scientific Research and Development (IJTSRD) ISSN: 2456-6470

Graph.4

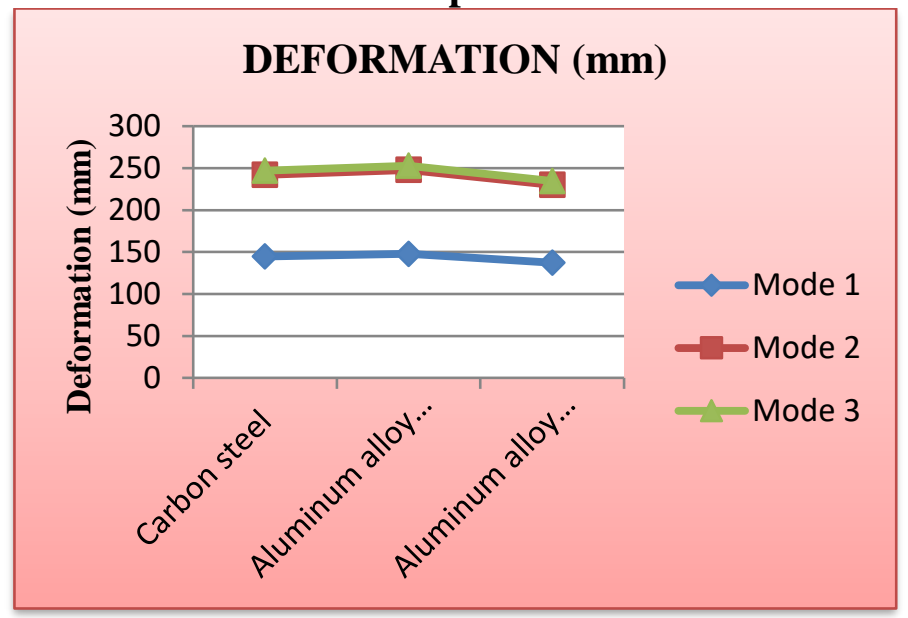

graph.5

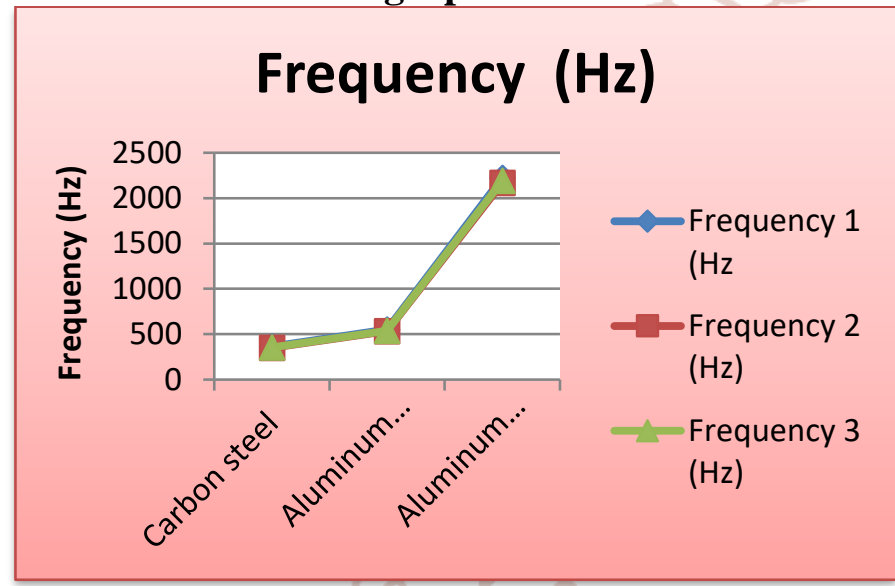

HARMONIC ANALYSIS

Table.3

\begin{tabular}{|c|c|c|c|}
\hline & $\begin{array}{c}\text { Deformati } \\
\text { on }(\mathrm{mm})\end{array}$ & Strain & $\begin{array}{c}\text { Stress } \\
(\mathrm{MPa})\end{array}$ \\
\hline Carbon steel & 0.0089825 & $4.6124 \mathrm{e}-5$ & 9.7985 \\
\hline $\begin{array}{c}\text { aluminum } \\
\text { alloy 7475 }\end{array}$ & 0.012063 & $6.1925 \mathrm{e}-5$ & 4.3415 \\
\hline $\begin{array}{c}\text { aluminum } \\
\text { alloy 6061 }\end{array}$ & 0.011382 & $5.8427 \mathrm{e}-5$ & 4.0147 \\
\hline
\end{tabular}

\section{Graph.6 DEFORMATION (mm)}

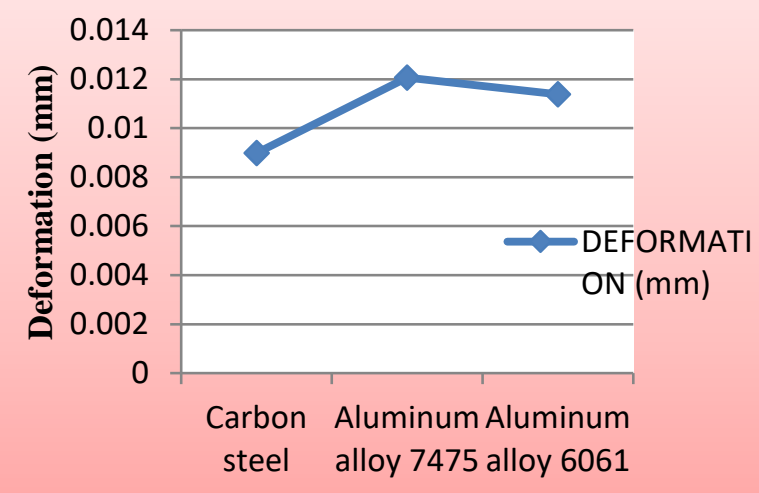

Graph.7

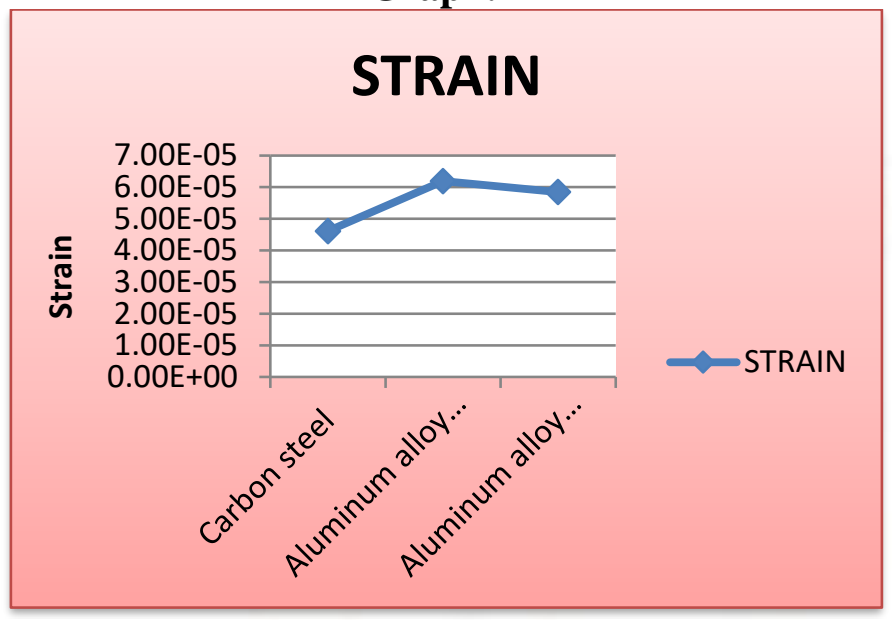

Graph.8

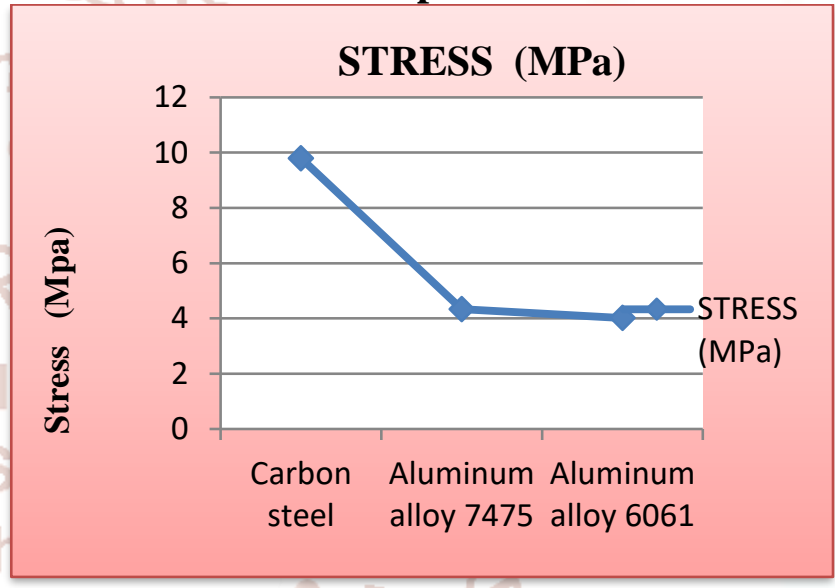

TRANSIENT ANALYSIS

Table.4 for1sec:

\begin{tabular}{|c|c|c|c|}
\hline & $\begin{array}{c}\text { Deformati } \\
\text { on }(\mathrm{mm})\end{array}$ & Strain & $\begin{array}{c}\text { Stress } \\
(\mathrm{MPa})\end{array}$ \\
\hline Carbon steel & 0.0012569 & 0.00016501 & 34.857 \\
\hline $\begin{array}{c}\text { aluminum } \\
\text { alloy 7475 }\end{array}$ & 0.0038504 & 0.0005039 & 34.89 \\
\hline $\begin{array}{c}\text { aluminum } \\
\text { alloy 6061 }\end{array}$ & 0.0039287 & 0.00051056 & 34.89 \\
\hline
\end{tabular}

Table.5 for 5sec:

\begin{tabular}{|c|c|c|c|}
\hline & $\begin{array}{c}\text { Deformati } \\
\text { On (mm) }\end{array}$ & Strain & $\begin{array}{c}\text { Stress } \\
(\mathrm{MPa})\end{array}$ \\
\hline Carbon steel & 0.0013719 & 0.0001801 & 38.045 \\
\hline $\begin{array}{c}\text { aluminum } \\
\text { alloy 7475 }\end{array}$ & 0.0042033 & 0.00054618 & 38.083 \\
\hline $\begin{array}{c}\text { aluminum } \\
\text { alloy 6061 }\end{array}$ & 0.0042888 & 0.00055728 & 38.083 \\
\hline
\end{tabular}


International Journal of Trend in Scientific Research and Development (IJTSRD) ISSN: 2456-6470

Table .6 for 10sec:

\begin{tabular}{|c|c|c|c|}
\hline & $\begin{array}{c}\text { Deformati } \\
\text { on }(\mathrm{mm})\end{array}$ & Strain & $\begin{array}{c}\text { Stress } \\
\text { (MPa) }\end{array}$ \\
\hline Carbon steel & 0.0014869 & 0.00019579 & 41.233 \\
\hline $\begin{array}{c}\text { aluminum } \\
\text { alloy 7475 }\end{array}$ & 0.0045558 & 0.00059194 & 41.274 \\
\hline $\begin{array}{c}\text { aluminum } \\
\text { alloy 6061 }\end{array}$ & 0.0046485 & 0.00060397 & 41.274 \\
\hline
\end{tabular}

\section{Graph.9}

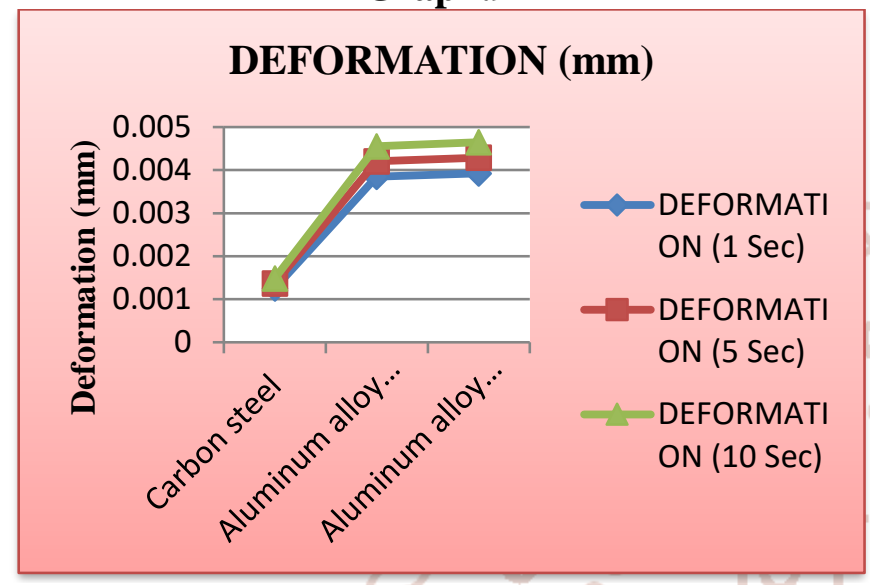

Graph.10

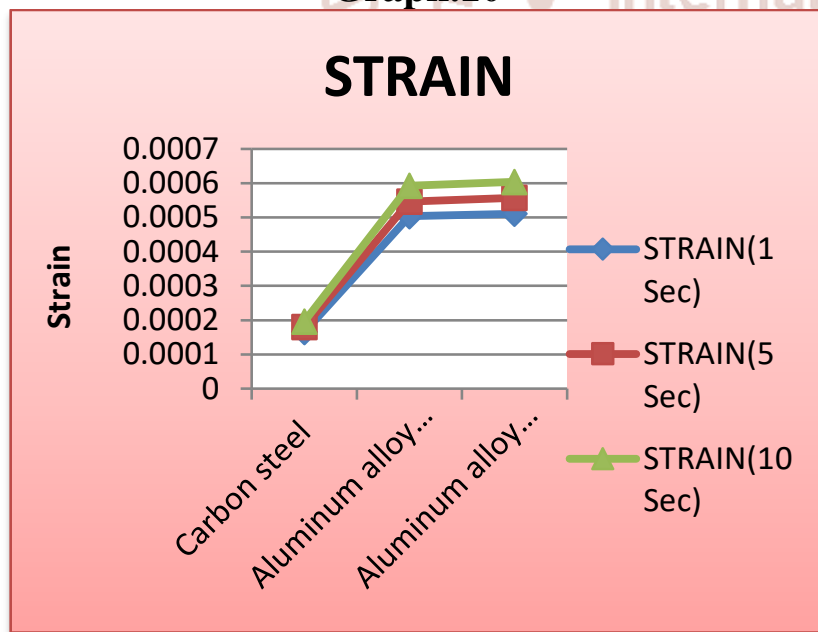

Graph.11

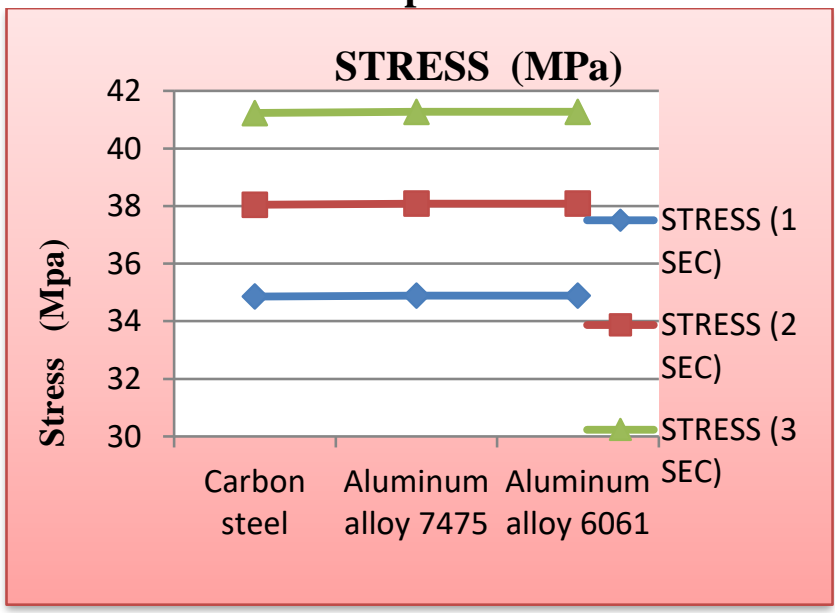

\section{CONCLUSION}

In this project we can modeled and designed 3D model of diesel engine connecting rod is by using software catia. Static, Modal, Harmonic and Transient analysis is performed on the connecting rod in ansys for different materials Structural Steel, Aluminum alloy 7475 and 6061 .

As we notice the plebiscite of structural analysis, for Aluminum alloy 7475 material the deformation and stress values are diminished. By noticing the plebiscite of modal analysis, the deformation values are less for Aluminum alloy. So vibrations will be less when Aluminum 7475 is used. By observing the plebiscite of Harmonic and Transient analysis, the stress values are diminishing for the Aluminum alloy 7475.

So it can be concluded that using Aluminum alloy 7475 is better due to less stress values and high strength to weight ratio.

\section{REFERENCES}

1. Static stress analysis of connecting rod using finite element approach by Abhinav Gautam, K Priya Ajit, IOSR Journal of Mechanical and Civil Engineering (IOSR-JMCE) e-ISSN: 2278-1684,pISSN: 2320-334X, Volume 10, Issue 1 (Nov. Dec. 2013), PP 47-51

2. Dynamic simulation of a connecting rod made of aluminum alloy using finite element analysis approach by Ram Bansal, IOSR Journal of Mechanical and Civil Engineering (IOSR-JMCE) e-ISSN: 2278-1684 Volume 5, Issue 2 (Jan. - Feb. 2013), PP 01-05

3. Analysis and optimization of connecting rod using AlFASiC composites by Kuldeep B, Arun L.R, Mohammed Faheem,ISSN: 2319-8753 International Journal of Innovative Research in Science, Engineering and Technology Vol. 2, Issue 6, June 2013

4. Connecting Rod Optimization for Weight and Cost Reduction by Pravardhan S. Shenoy and Ali Fatemi, SAE Technical Paper 2005-01-0987, 2005, doi:10.4271/2005-01-0987.

5. Process capability improvement of an engine connecting rod machining process by GVSS Sharma and P Srinivasa Rao, Journal of Industrial Engineering International 2013, 9:37 doi:10.1186/2251-712X-9-37 
6. Modeling and Analysis of Two Wheeler Connecting Rod by K. Sudershn Kumar, Dr.K. Tirupathi Reddy, Syed Altaf Hussain, International Journal of Modern Engineering Research (IJMER), Vol.2, Issue.5, Sep-Oct. 2012 pp-3367-3371 ISSN: 2249-6645

7. Design Evaluation and Optimization of Connecting Rod Parameters Using FEM by Suraj Pal, Sunil kumar, International Journal of Engineering and Management Research, Vol.-2, Issue-6, December 2012
8. Stress Analysis of I.C.Engine Connecting Rod by FEM and Photo elasticity by Prof. Vivek C. Pathade , Dr. Dilip S. Ingole, IOSR Journal of Mechanical and Civil Engineering (IOSR-JMCE) e-ISSN: 2278-1684 Volume 6, Issue 1 (Mar. Apr. 2013), PP 117-125

9. Design And Finite Element Analysis Of Aluminium-6351 Connecting Rod by Priyank D. Toliya, Ravi C. Trivedi, Prof. Nikhil J. Chotai, Volume/Issue: Vol.2 - Issue 5 (May - 2013), eISSN: 2278-0181

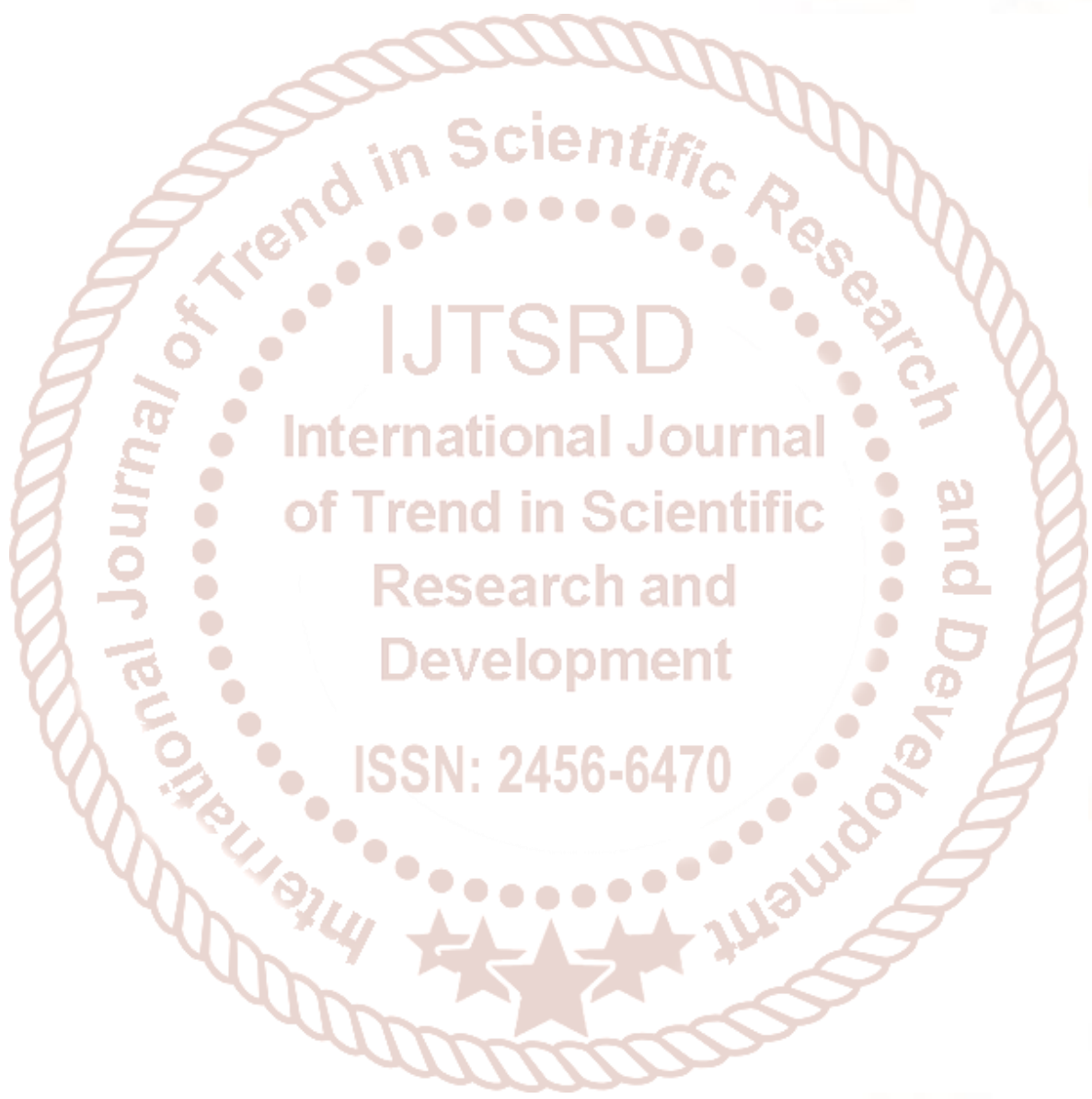

